Contact Dr Kerri Basile.

E-mail: kerri.basile@ health.nsw.gov.au

1. Australian Government Department of Health. Coronavirus (COVID19) current situation and case numbers. Cited 20 Sep 2020. https://www. health.gov.au/news/health-alerts/novel-coronavirus-2019-ncov-healthalert/coronavirus-covid-19-current-situation-and-case-numbers\#testsconducted-and-results.

2. Therapeutic Goods Association. COVID-19 testing in Australia - information for health professionals. COVID-19 test performance. Cited 17 Jun 2020. https://www.tga.gov.au/covid-19-testing-australia-information-health-professionals

3. Woloshin S, Patel N, Kesselheim AS. False negative tests for SARS-CoV 2 infection - challenges and implications. N Engl J Med 2020; 383: e38.

4. US Food and Drug Administration. Emergency Use Authorization (EUA) information, and list of all current EUAs. Cited 17 Jun 2020. https://www.fda.gov/emergency-preparedness-and-response/mcm-legalregulatory-and-policy-framework/emergency-use-authorization

5. Australian Government Department of Health. PHLN guidance on laboratory testing for SARS-CoV-2 (the virus that causes COVID-19). Cited 8 Jun 2020. https://www.health.gov.au/resources/publications/ phln-guidance-on-laboratory-testing-for-sars-cov-2-the-virus-thatcauses-covid-19

6. Australian Government Department of Health. PHLN guidance on nucleic acid test result interpretation for SARS-CoV-2. Cited 19 Sep 2020. https://www health gov au/resources/publications/phln-guidanceon-nucleic-acid-test-result-interpretation-for-sars-cov-2

7. COVID-19 National Incident Room Surveillance Team. COVID-19, Australia: Epidemiology Report 9 (Reporting week to 23:59 AEDT 29 March 2020). Commun Dis Intell 2020; 44: https://doi.org/10.33321/ cdi.2020.44.29.

8. Rahman H, Carter I, Basile K, et al. Interpret with caution: an evaluation of the commercial AusDiagnostics versus in-house developed assays for the detection of SARS-CoV-2 virus. J Clin Virol 2020; 127: 104374.

9. Bustin SA, Nolan T. RT-qPCR testing of SARS-CoV-2: a primer. Int $J$ Mol Sci 2020; 21: E3004.

10. US Food and Drug Administration. SARS-CoV-2 reference panel comparative data. Cited 21 Sep 2020. https://www.fda.gov/medicaldevices/coronavirus-covid-19-and-medical-devices/sars-cov-2reference-panel-comparative-data

11. European Centre for Disease Prevention and Control. Threat Assessment Brief. Reinfection with SARS-CoV: considerations for public health response. 21 Sep 2020. https://www.ecdc.europa.eu/sites/default/files/ documents/Re-infection-and-viral-shedding-threat-assessment-brief.pdf

DOI: https://doi.org/10.1016/j.pathol.2020.09.009

\section{The impact of viral transport media on PCR assay results for the detection of nucleic acid from SARS-CoV-2}

Sir,

For several decades a variety of medium solutions have been recommended to stabilise specimens for the detection of bacteria and viruses, particularly during diagnostic investigations. These have usually been based on balanced salt or saline solutions with a buffering capacity to maintain a 'near-neutral' $\mathrm{pH}$. To enhance the stability of viruses a spectrum of protein supplements has and continues to be recommended. ${ }^{1-3}$ While some laboratories have prepared viral transport medium (VTM) 'in house', commercial preparations are used extensively and are often supplied as part of a sample collection kit with sterile swabs. Testing of samples by cultural methods meant that the emphasis of studies for the evaluation of these products originally focussed on the capacity of a preparation to maintain the infectivity of viruses at different temperatures while being held prior to and during transport and while being stored at the laboratory. With the widespread introduction of molecular based diagnostic assays, especially real time PCR (qPCR), studies have been undertaken to evaluate the stability of viruses in VTMs, particularly in commercially prepared products, while being held at a range of temperatures. ${ }^{4,5}$ However, while thermal stability has been considered, generally little attention has been given to other characteristics of the VTM or the potential impact of endogenous components. One commercially available product is specifically designed to inactivate viruses and bacteria and contains components to inhibit the activity of nucleases that may be present in the sample. ${ }^{6}$ There are some other products that are recommended for use in molecular detection assays but the manufacturers provide no comment that these products are unlikely to be suitable for samples where virus culture will be attempted.

During large-scale disease epidemics there can be pressure placed on the capacity of manufacturers to supply transport media and, during a pandemic, supply-chain and manufacturing pressures can become prohibitive. During the current (2020) SARS-CoV-2 pandemic, there has been an acute shortage of VTM in Australia because of a combination of both local and international demand, the lack of a local manufacturer and partly because of reduced international airline flights to Australia. Consequently, many different VTMs and similar solutions have been used to meet the demand for transport media generated by large scale diagnostic and surveillance testing. After becoming aware of concerns of variable results for the same samples in different assays, we initiated a study to compare the stability of SARSCoV-2 RNA in several commercially manufactured VTMs and an in-house product. The commercial products were UTM-RT (Copan, Italy), Citoswab (Citotest Labware, China) and CP VTF (Edwards, Australia), while the in-house product (VTM-1) was based on phosphate buffered saline (PBS $\mathrm{pH}$ 7.2) supplemented with $0.5 \%$ gelatin (PBGS). The commercial products are believed to be supplemented with bovine serum albumen and gelatin or bovine serum. Sterile phosphate buffered saline (PBS), $\mathrm{pH} 7.2$, was used as a control. Within 30-45 minutes of preparation, the dilutions of RNA in each VTM were extracted and tested by semiquantitative real time PCR (qRT-PCR). Subsequently, this pilot experiment was repeated under the same conditions with a series of dilutions of a high titred patient sample (P66) which had been recently collected and with a Type A influenza virus and the RNA extracted from it.

Total nucleic acid was extracted from $50 \mu \mathrm{L}$ of each sample with a magnetic bead-based viral RNA extraction kit (MagMax96 Viral RNA; Ambion, USA) run on a Kingfisher96 magnetic particle handling system (ThermoFisher, USA). The nucleic acid was eluted in $50 \mu \mathrm{L}$ and $5 \mu \mathrm{L}$ run in an in house qRT-PCR. Primers and probes were directed at the SARS-CoV-2 E gene ${ }^{7}$ and the RdRp gene. ${ }^{8}$ These SARS$\mathrm{CoV}-2$ primers and probes were used in a triplex assay with the inclusion of an exogenous RNA internal control (XIPC) assay. ${ }^{9}$ This XIPC RNA (approximately 80 copies/ $\mu \mathrm{L})$ was included in the sample lysis buffer prior to the extraction of nucleic acid. A commercial reverse transcriptase mastermix (AgPath-ID One-Step RT-PCR kit; Life Technologies, USA) was used for the qRT-PCR and run on an ABI7500 or Quantstudio 5 (ThermoFisher Scientific, USA) thermocycler. The design of this study and the methods employed are described in full elsewhere. ${ }^{10}$ A selection of 
samples was also tested using the CDC designed assay (2019nCoV_N2) targeting the $\mathrm{N}$ gene. ${ }^{11}$

This investigation showed that there was a profound impact on the detection of SARS-CoV-2 RNA after dilution in the commercial VTM products (VTM-2 to VTM-4). SARS-CoV-2 RNA was not detected in any dilution prepared in VTM-2 and very weak reactivity (approaching the assays limit of detection) was observed for VTM-3 and VTM-4 in the sample with the highest concentration of RNA (Table 1). In contrast, samples diluted in PBS and VTM-1 gave almost identical results, with a $\mathrm{Ct}$ value of approximately 21 for the highest RNA concentration. This difference between the PBS/VTM-1 result and the results for the other VTMs represents a reduction in analytical sensitivity of approximately $6 \log _{10}$ for the detection of free SARS-CoV-2 RNA. The results for the XIPC were highly reproducible and similar for each dilution in each solution, confirming the high efficiency of RNA extraction and no apparent impact of PCR inhibitors.

Testing was then undertaken to determine whether there might be a reduction in sensitivity when testing a sample that presumptively contains high quality intact virions. In this instance, the results for the dilutions of virus (Table 2) were similar for each VTM and the PBS, as were the results for the XIPC. Similar results for both SARS-CoV-2 purified RNA and the patient sample were also obtained in the CDC assay (2019-nCoV_N2) targeting the $\mathrm{N}$ gene.

The experiments undertaken have shown that some of the VTM solutions examined had a significant and deleterious impact on purified viral RNA. The synthetic XIPC RNA that was used throughout this study was not immediately affected because it was prepared in a tRNA solution and included in the sample lysis buffer which includes inhibitors of nuclease activity. Further, the nucleic acid is extracted from the sample immediately after addition of the lysis buffer. However, to confirm that the XIPC construct could be affected by components in the VTMs under study, two concentrations of XIPC were prepared in PBS and in each of the VTM solutions and were tested after being held at room temperature for 1 or 48 hours. The adverse effect of each commercial VTM was apparent within the first hour and no RNA was detected after 48 hours, whereas the results for XIPC held in PBS or VTM-1 did not change.

Finally, to demonstrate that the adverse effects of the commercial VTM solutions were not restricted to SARSCoV-2 RNA, a similar experiment was undertaken with RNA from a Type A influenza virus. Similar results were obtained (Table 3), with no viral RNA detected after dilution in each of the commercial VTM solutions but without any change in the RNA concentrations after dilution in the inhouse VTM or PBS.

The results of these studies clearly indicate that the commercially prepared VTM solutions have had an adverse impact on the ability to detect both SARS-CoV-2 and influenza RNA. The results for the RNA preparations diluted in the commercial VTMs would suggest that there are components of these VTMs that have prevented the detection of the RNA in these samples. As the detection of the XIPC was not

Table 1 Comparison of SARS-CoV-2 qRT-PCR Ct values when testing dilutions of purified SARS-CoV-2 RNA in different viral transport media

\begin{tabular}{|c|c|c|c|c|c|c|c|c|c|c|}
\hline \multirow[t]{2}{*}{ Dilution of SARS-CoV-2 RNA } & \multicolumn{2}{|c|}{ PBS } & \multicolumn{2}{|c|}{ VTM-1 } & \multicolumn{2}{|c|}{ VTM-2 } & \multicolumn{2}{|c|}{ VTM-3 } & \multicolumn{2}{|c|}{ VTM-4 } \\
\hline & Target & XIPC & Target & XIPC & Target & XIPC & Target & XIPC & Target & XIPC \\
\hline 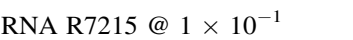 & 20.9 & 28.4 & 20.8 & 28.2 & - & 30.1 & 39.4 & 31.1 & 39.8 & 30.6 \\
\hline RNA R7215@1 @ $1 \times 10^{-2}$ & 24.3 & 29.7 & 24.3 & 29.6 & - & 30.5 & - & 30.9 & - & 30.4 \\
\hline RNAR7215@1 @ $10^{-3}$ & 25.8 & 30.5 & 25.6 & 30.0 & - & 30.5 & - & 30.3 & - & 30.7 \\
\hline RNAR7215@1/4 × $10^{-3}$ & 29.4 & 30.3 & 30.3 & 30.2 & - & 30.3 & - & 29.9 & - & 28.8 \\
\hline RNAR7215@1/8 × $10^{-3}$ & 31.8 & 29.5 & 31.3 & 28.3 & - & 29.5 & - & 30.7 & - & 30.2 \\
\hline RNAR7215@1/16 × $10^{-3}$ & 32.5 & 29.8 & 31.6 & 28.7 & - & 30.0 & - & 30.5 & - & 29.3 \\
\hline RNAR7215@1/32 × 10 & 33.8 & 29.9 & 34.6 & 29.0 & - & 30.8 & - & 30.5 & - & 30.9 \\
\hline RNAR7215@1/64 × $10^{-3}$ & 40.2 & 29.9 & 40.3 & 28.4 & - & 30.6 & - & 29.0 & - & 29.1 \\
\hline RNAR7215@1/128 × $10^{-3}$ & 40.3 & 27.9 & 40.8 & 30.0 & - & 29.0 & - & 29.6 & - & 30.4 \\
\hline
\end{tabular}

- , not detected.

Table 2 Comparison of SARS-CoV-2 qRT-PCR Ct values when testing a patient sample diluted in different viral transport media

\begin{tabular}{|c|c|c|c|c|c|c|c|c|c|c|}
\hline \multirow[t]{2}{*}{ Dilution of patient sample } & \multicolumn{2}{|c|}{ PBS } & \multicolumn{2}{|c|}{ VTM-1 } & \multicolumn{2}{|c|}{ VTM-2 } & \multicolumn{2}{|c|}{ VTM-3 } & \multicolumn{2}{|c|}{ VTM-4 } \\
\hline & Target & XIPC & Target & XIPC & Target & XIPC & Target & XIPC & Target & XIPC \\
\hline P-66@1 @ $1 \times 10^{-2}$ & 23.7 & 29.7 & 24.1 & 29.9 & 24.2 & 30.0 & 25.0 & 30.3 & 24.5 & 29.5 \\
\hline P-66@1 @ $1 \times 10^{-3}$ & 27.3 & 29.8 & 27.6 & 30.2 & 28.6 & 30.6 & 29.0 & 30.6 & 27.8 & 30.4 \\
\hline P-66@1 @ $1 \times 10^{-4}$ & 28.0 & 29.6 & 29.9 & 31.0 & 32.2 & 30.1 & 32.7 & 31.2 & 31.0 & 30.4 \\
\hline $\mathrm{P}-66 @ 1 / 2 \times 10^{-4}$ & 32.6 & 30.0 & 32.8 & 29.9 & 33.1 & 30.0 & 33.0 & 30.0 & 33.5 & 30.8 \\
\hline P-66@1/8 × $10^{-4}$ & - & 29.5 & - & 30.6 & - & 30.4 & - & 29.9 & - & 30.7 \\
\hline P-66@1/16 × $10^{-4}$ & - & 30.8 & 40.1 & 30.2 & - & 30.6 & - & 30.2 & - & 31.0 \\
\hline P-66@1/32 × $10^{-4}$ & - & 30.5 & - & 30.8 & - & 30.6 & - & 30.3 & - & 30.3 \\
\hline P-66@1/64 × $10^{-4}$ & - & 29.6 & - & 30.5 & - & 29.9 & - & 31.0 & - & 30.3 \\
\hline
\end{tabular}

- , not detected. 
Table 3 Comparison of Type A influenza qRT-PCR Ct values when testing purified influenza virus RNA diluted in different viral transport media

\begin{tabular}{|c|c|c|c|c|c|c|c|c|c|c|}
\hline \multirow[t]{2}{*}{ Dilution of influenza viral RNA } & \multicolumn{2}{|c|}{ PBS } & \multicolumn{2}{|c|}{ VTM-1 } & \multicolumn{2}{|c|}{ VTM-2 } & \multicolumn{2}{|c|}{ VTM-3 } & \multicolumn{2}{|c|}{ VTM-4 } \\
\hline & Target & XIPC & Target & XIPC & Target & XIPC & Target & XIPC & Target & XIPC \\
\hline 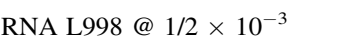 & 30.7 & 29.2 & 30.8 & 29.6 & - & 29.4 & - & 29.5 & - & 28.9 \\
\hline RNA L998@1/4 × 10 & 31.7 & 29.5 & 31.3 & 29.5 & - & 29.1 & - & 29.1 & - & 29.6 \\
\hline RNA L998@1/8 × $10^{-3}$ & 34.0 & 29.9 & 33.6 & 30.1 & - & 29.8 & - & 29.7 & - & 29.6 \\
\hline RNA L998@1/16 × $10^{-3}$ & 34.5 & 29.6 & 34.0 & 29.5 & - & 29.7 & - & 29.7 & - & 29.2 \\
\hline RNA L998@1/64 × $10^{-3}$ & - & 29.3 & 37.5 & 29.5 & - & 29.5 & - & 29.2 & - & 29.4 \\
\hline
\end{tabular}

- , not detected.

affected, we propose that these results provide evidence of the presence of a nuclease(s) in these VTMs rather than a component that may affect the efficiency of nucleic acid extraction or the PCR assay. The impact on these samples was rapid as all samples were extracted within one hour of preparation, yet no RNA was detected in a sample that was estimated to contain more than 3000 copies of viral RNA in a $5 \mu \mathrm{L}$ sample. The same result was obtained with assays that were directed at three different regions of the SARS-CoV-2 genome. The results obtained for the virus samples that were diluted to similar concentrations as the RNA samples and held for the same time period also support this hypothesis because it is expected that these samples contained a high proportion of intact nucleocapsids that offered protection to the viral RNA. The same trends were observed with the influenza A virus RNA and presumptively intact virions.

It is also important to recognise that these observations reflect the outcome of contact between viral nucleic acid and VTM for less than 1 hour in each instance. The levels of nucleic acid that were destroyed almost immediately after addition to the VTMs were not insignificant. The change in concentration of RNA from a Ct value of approximately 21 in both PBS and VTM-1 to being undetectable in the other VTM solutions represents a reduction of approximately ten thousand-fold and cannot be ignored. While it might be argued that the adverse impact on whole virus appeared to be slight, free nucleic acid and perhaps whole virus was destroyed at levels that could be of diagnostic relevance. ${ }^{12}$ Unfortunately, more extensive testing of patient samples was not possible because each patient sample that was submitted to the laboratory had already been exposed to an individual VTM for many hours. Deleterious effects may have already commenced during this period. To undertake a genuine comparison of patient samples in different VTM, it would be necessary to immerse the swab into a 'neutral' solution such as PBS for a short period then divide into aliquots for addition to each of the VTM solutions under study.

While the impact on RNA viral genomes is likely to be markedly greater than on DNA sequences due to the presence of ribose which is more susceptible to degradation, the outcome cannot be predicted as secondary structure may also have an influence. ${ }^{12}$ The speed and severity of the impact may also vary depending on the nucleic acid target, as shown by the differences between the results for the two RNA viruses and the XIPC. Further, it cannot be assumed that the target nucleic acid will always be protected by nucleoprotein. Degradation will occur during the course of an infection and also under conditions where sample collection, transport and storage are sub-optimal. Additionally, the adverse effects observed in this study could potentially be exacerbated with alternative nucleic acid purification technologies that take longer than the 20 minutes required for the magnetic-bead based method used in the current study. While undertaking surveillance and epidemiological tracing during a pandemic, failure to detect a moderate level of RNA in a person who is asymptomatic could result in a critical source of infection remaining undetected. However, with the selection of an appropriate transport medium, nucleic acid degradation, even at room temperature, can be minimal. This is clearly shown by the performance of the in-house medium (VTM-1) where there was little evidence of deterioration of the RNA samples after holding at room temperature for 2 days. Further, there was no significant deterioration of virus from patient samples when held in VTM- 1 at $4^{\circ} \mathrm{C}$ for more than 6 weeks. Unfortunately there was insufficient material to investigate the longer term stability in the commercial VTMs under these conditions.

The World Health Organization, the US Centers for Disease Control and Prevention (CDC) and the UK government have each provided recommendations for the formulation of VTM solutions to be used for the collection of specimens for SARS-CoV-2 testing and comment that a supplement of protein or glycerol should be added to enhance the stability of viruses. ${ }^{1-3}$ We believe that, while this is an essential feature of a high quality VTM, it is in achieving this requirement that the current problem with the commercially available VTMs may have arisen. Our in-house VTM includes gelatin which is extracted from animal tissues by treatment at very low or high $\mathrm{pH}$, and prolonged boiling at high temperatures before sterilisation and drying. These steps inactivate both enzymes and infectious agents that are present as well as destroying residual nucleic acid. Further, during the preparation of VTM-1, the PBS solution to which the gelatin has already been added is also sterilised by heat treatment. In contrast, products that include bovine serum albumen or other serumderived components, such as VTMs 2, 3 and 4, cannot be sterilised by autoclaving without coagulation of the protein supplement. Therefore, for these VTMs, the raw materials must each be free of nucleases and proteinases prior to sterilisation by methods that do not include heating.

As indicated in the UK government guideline, ${ }^{3}$ there is a clear need for the 'Use (of) alternative swabs and transport medium in accordance with a locally validated laboratory strategy' to demonstrate fitness for purpose. The specifications for products that might be used for both nucleic acid detection methods and virus culture are likely to be more rigorous than for those VTMs that are only used for one laboratory method. The special requirements for products that 
are suitable for nucleic acid testing have been recognised by some manufacturers who have developed specific transport media to inactivate the viruses of interest and to minimise the degradation of nucleic acid. ${ }^{6}$ Some of the major manufacturers of VTM solutions also offer products with additives to reduce nuclease activity but most of these also preclude opportunities to undertake virus culture. However, these limitations are often not clearly disclosed and may not be apparent to purchasing departments, especially during a pandemic, when any VTM may be mistakenly thought 'fit for purpose'.

In conclusion, the results of this study provide examples of how the composition of a VTM could have an impact on the outcome of nucleic acid based testing and, in particular, situations where either there is a need to detect RNA that is not packaged into a nucleocapsid or where RNA constructs may be diluted in a VTM for use as a positive control in an assay or perhaps for proficiency testing. Finally, and particularly in the face of a pandemic, users should be reminded that products fit for one purpose may not be suitable for an alternative use. A product that may be eminently suitable for virus culture purposes could result in misleading results if used for nucleic acid-based tests.

Acknowledgements: The authors are indebted to Mr Ian Carter, Institute of Clinical Pathology and Medical Research, Westmead Hospital, for the generous supply of the purified SARS-CoV-2 RNA and for referral of the patient sample. We also appreciate the productive discussions with Drs Catherine Pitman and Dominic Dwyer, NSW Health Pathology, regarding the need for rigorous evaluation of viral transport media. We also thank Dr Deb Finlaison for helpful comments on the draft manuscript and Shannon Mollica, Rodney Davis and other staff of the Virology Laboratory at EMAI for their assistance during the preparation of samples for the initial experiment and the longer-term storage of swabs.

Conflicts of interest and sources of funding: Financial support for the conduct of this research and for preparation of the article was provided to the authors by the NSW Department of Primary Industries (NSWDPI) during the course of their employment. NSW DPI played no role in the planning or conduct of the research nor in the decision to submit this paper for publication. The authors state that there are no conflicts of interest to disclose.

\section{P. D. Kirkland, M. J. Frost}

Virology Laboratory, Elizabeth Macarthur Agriculture Institute, Menangle, NSW, Australia

Contact Dr P. D. Kirkland.

E-mail: peter.kirkland@dpi.nsw.gov.au

1. World Health Organization (WHO). WHO Manual on Animal Influenza Surveillance and Diagnosis. WHO/CDS/CSR/NCS/2002.5. Geneva: WHO, 2002. https://apps.who.int/iris/handle/10665/68026

2. Centers for Disease Control and Prevention. Preparation of viral transport medium. Standard Operating Procedure SOP\#: DSR-052-02. Cited 30 May 2020. https://www.cdc.gov/coronavirus/2019-ncov/downloads/ Viral-Transport-Medium.pdf

3. UK Government. COVID-19: Guidance for Sampling and for Diagnostic Laboratories. Cited 30 May 2020. https://www.gov.uk/ government/publications/wuhan-novel-coronavirus-guidance-forclinical-diagnostic-laboratories
4. Druce J, Garcia K, Tran T, et al. Evaluation of swabs, transport media and specimen transport conditions for optimal detection of viruses by PCR. J Clin Microbiol 2012; 50: 1064-5.

5. Vermeiren C, Marchand-Senécal X, Sheldrake E, et al. Comparison of Copan Eswab and FLOQswab for COVID-19 PCR diagnosis: working around a supply shortage. J Clin Microbiol 2020; 58: e00669-20.

6. Daum LT, Worthy SA, Yim KC, et al. A clinical specimen collection and transport medium for molecular diagnostic and genomic applications. Epidemiol Infect 2011; 139: 1764-73.

7. Corman VM, Landt O, Kaiser M, et al. Detection of 2019 novel coronavirus (2019-nCoV) by real-time RT-PCR. Euro Surveill 2020; 25: 2000045.

8. World Health Organization. Protocol: Real-time RT-PCR Assays for the Detection of SARS-CoV-2. Paris: Institut Pasteur, 2020. https://www. who.int/docs/default-source/coronaviruse/real-time-rt-pcr-assays-for-thedetection-of-sars-cov-2-institut-pasteur-paris.pdf?sfvrsn=3662fcb6 2

9. Schroeder ME, Bounpheng MA, Rodgers S, et al. Development and performance evaluation of calf diarrhea pathogen nucleic acid purification and detection workflow. J Vet Diagn Invest 2012; 24: 945-53.

10. Kirkland PD, Frost MJ. 2020. The impact of viral transport media on PCR assay results for the detection of nucleic acid from SARS-CoV-2 and other viruses. BioRxiv 2020; Jun 10: https://doi.org/10.1101/ 2020.06.09.142323.

11. Centers for Disease Control and Prevention. 2019-Novel Coronavirus (2019-nCoV) Real-Time rRT-PCR Panel Primers and Probes. Cited 30 May 2020. https://www.who.int/who-documents-detail/molecularassays-to-diagnose-covid-19-summary-table-of-available-protocols

12. Relova D, Rios L, Acevedo AM, et al. Impact of RNA degradation on viral diagnosis: an understated but essential step for the successful establishment of a diagnosis network. Vet Sci 2018; 5: 19.

DOI: https://doi.org/10.1016/j.pathol.2020.09.013

\section{Contamination of SARS-CoV-2 RT- PCR probes at the oligonucleotide manufacturer}

Sir,

Severe acute respiratory syndrome coronavirus 2 (SARSCoV-2) was first identified in Wuhan, China, in December 2019 as the aetiological agent of Coronavirus disease 2019 (COVID-19). ${ }^{1,2}$ Since then, the disease has spread rapidly worldwide and the World Health Organization (WHO) declared a pandemic on 11 March $2020 .^{3,4}$ At the beginning of the outbreak, rapid development and implementation of reliable detection methods became an immediate priority for clinical laboratories worldwide, and reverse transcription polymerase chain reaction (RT-PCR) methods, including those provided by the WHO, ${ }^{5,6}$ have been implemented broadly. At the early stage of the outbreak, however, positive control material for RT-PCR assays (from positive patient samples, or viral culture) were not readily available. In such circumstances laboratories often turn to using synthetic controls (synDNA fragments or plasmids). ${ }^{7,8}$ These synthetic controls have their advantages, particularly in that the controls can be acquired as readily as PCR primers and probes. Yet, depending on how they are designed, precautions must be taken when handling such controls as trace amounts of this material can potentially cause contamination in the same way as that caused by PCR products. Here we report contamination of a SARS-CoV-2 probe that our evidence suggests occurred at the oligonucleotide manufacturer, and was due to the manufacturer synthesising full length control oligonucleotides (spanning from the forward to reverse primers) in parallel with our probe orders. 